\section{APPLES, ORANGE, AND PEARS. TRANSCATHETER AORTIC VALVE IMPLANTATION IN INTERMEDIATE RISK PATIENTS HAS BORNE FRUIT}

\section{To the Editor:}

Transcatheter aortic valve implantation (TAVI) has become a valid alternative in patients who are at high risk for surgical mortality or who are not suitable candidates for surgery. There has also been interest in exploring TAVI's potential role in patients at intermediate risk. Recently published Placement of Aortic Transcatheter Valves (PARTNER) 2 trial randomly assigned 2032 patients at intermediate risk to undergo TAVI with SAPIEN XT valve (Edwards Lifesciences Corporation, Irvine, Calif) or surgical replacement. ${ }^{1}$ The treatments were comparable in terms of the composite end point of death from any cause or disabling stroke at 2 years. Surgery was still associated with fewer major vascular complications and lower incidence of paravalvular aortic regurgitation. ${ }^{1}$

The same authors ${ }^{2}$ simultaneously published 1-year outcomes of an observational cohort of patients at intermediate risk undergoing TAVI with a new modified system (SAPIEN 3; Edwards) and compared these results with those of patients undergoing surgical aortic valve replacement in the PARTNER 2 trial by using an approach based on propensity score (PS) analysis. TAVI with SAPIEN 3 was found to be superior to surgical replacement and has been advocated as a preferred alternative in this group. This comparison, however, presents several methodologic flaws that merit attention.

The major challenge for observational studies is the inherent vulnerability to selection bias. Despite statistical adjustment, including PS, apples and orange will never be exactly the same. ${ }^{3}$ Moreover, the crucial assumption of PS-based analysis is that the treatment assignment should be strongly ignorable. ${ }^{4}$ This simply means that to be compared, both treatments should be potentially adoptable in the same subject. In their study, Thourani and colleagues ${ }^{2}$ violated this basic principle, because subjects in the TAVI group (pears in this case) were not exposed to the surgical allocation because of the "strategic case selection" for TAVI.

To prove the superiority of the new SAPIEN 3, Thourani and colleagues ${ }^{2}$ made use of the past: enrolment periods of the compared cohorts were different (mainly 2011 and 2012 for the surgical replacement cohort and 2014 for the SAPIEN 3 cohort). This difference may have translated into different patterns of practice, including improvement in case selection with time.

Many patients were excluded from the original SAPIEN 3 cohort because no echocardiographic follow-up at 1 year was available $(165 / 936 ; 18 \%)$. It is well known that patients who are unavailable for complete follow-up are more likely to have adverse events, thus underestimating the rate of adverse events in the TAVI group.

The surgical replacement group was defined according to the as-treated principle. It thus included the higher risk subgroup of patients initially allocated to TAVI who underwent surgery instead, which also may have biased results in favor of the TAVI group. ${ }^{5}$

The criteria for treatment selection in the PARTNER 2 trial was a Society of Thoracic Surgeons score of at least $4.0 \%$, but an upper limit was not prespecified. In contrast, in the SAPIEN 3 cohort, the Society of Thoracic Surgeons score upper limit was set to $8 \%$. Consequently, patients at higher risk were enrolled in the PARTNER 2 trial, as demonstrated by a statistically significant difference in Society of Thoracic Surgeons score between the groups $(P=.0002)$ and differences in other variables.

More simply, one might argue that the main change in the SAPIEN 3 is an external skirt to prevent paravalvular regurgitation, and it is highly unlikely that a few minor changes translated into the significant improvement seen in such hard clinical end points as mortality at 30 days (from $6.1 \%$ to $1.1 \%$ ).

In conclusion, results reported by Thourani and colleagues ${ }^{2}$ are biased by different practice patterns that affected treatment decisions and outcomes. Regardless of sophisticated statistical adjustment, observational studies should always be intended as hypothesis generating in the case of lack of evidence from randomized studies rather than as hypothesis testing.

\footnotetext{
The Editor welcomes submissions for possible publication in the Letters to the Editor section that consist of commentary on an article published in the Journal or other relevant issues. Authors should: • Include no more than 500 words of text, three authors, and five references. $\bullet$ Type with double-spacing. • See http://jtcs.ctsnetjournals.org/ misc/ifora.shtml for detailed submission instructions. • Submit the letter electronically via jtcvs.editorialmanager.com. Letters commenting on an article published in the JTCVS will be considered if they are received within 6 weeks of the time the article was published. Authors of the article being commented on will be given an opportunity of offer a timely response ( 2 weeks) to the letter. Authors of letters will be notified that the letter has been received. Unpublished letters cannot be returned.
} 
Authors have nothing to disclose with regard to commercial support.

\section{References \\ 1. Leon MB, Smith CR, Mack MJ, Makkar RR, Svensson LG, Kodali SK, et al; PARTNER 2 Investigators. Transcatheter or surgical aortic-valve replacement in intermediate-risk patients. $N$ Engl J Med. 2016;374:1609-20. \\ 2. Thourani VH, Kodali S, Makkar RR, Herrmann HC, Williams M, Babaliaros V, et al. Transcatheter aortic valve replacement versus surgical valve replacement in intermediate-risk patients: a propensity score analysis. Lancet. 2016;387: 2218-25. \\ 3. Baser O. Too much ado about propensity score models? Comparing methods of propensity score matching. Value Health. 2006;9:377-85. \\ 4. Austin PC. An introduction to propensity score methods for reducing the effects of confounding in observational studies. Multivariate Behav Res. 2011;46: 399-424. \\ 5. Gravel J, Opatrny L, Shapiro S. The intention-to-treat approach in randomized controlled trials: are authors saying what they do and doing what they say? Clin Trials. 2007;4:350-6.}

http://dx.doi.org/10.1016/j.jtcvs.2016.10.043

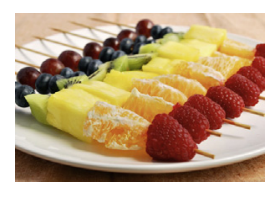

TRANSCATHETER

AORTIC VALVE

REPLACEMENT IN

INTERMEDIATE-RISK PATIENTS

\section{Reply to the Editor:}

We appreciate the letter by Benedetto and Uva regarding our study comparing use of the Sapien 3 Transcatheter Aortic Valve (TAV) (Edwards Lifesciences, Inc, Irvine, Calif) in intermediate-risk patients with results of patients undergoing surgery in the Placement of Aortic Transcatheter Valves 2 randomized trial. ${ }^{1,2}$

The authors are completely correct that a propensity score analysis does not replace or compare with a true randomized trial, although the authors have commonly used propensity score matching in their own research endeavors. Furthermore, we agree that observational studies by nature are more prone to selection bias and cannot be equated to randomized trials. However, propensity score analysis is not trying to make apples into oranges or compare them, rather it is trying to make 2 fruit salad bowls have roughly equal proportions of apples, oranges, and pears so that they may taste similar and be comparable to a discerning palate. The authors note that the major challenge to observational studies is their vulnerability to selection bias and that despite statistical adjustments, including propensity scores (ie, analysis), apples and oranges will never be the same. The authors note that the 2 enrollment periods of the 2 cohorts were different (mainly 2011 and 2012 for the surgical aortic valve replacement [SAVR] cohort and 2014 for the Sapien 3 cohort), and this might have translated into different patterns of practice, including improvement in case selection. We argue that absolutely nothing changed in the performance of SAVR from 2012 to 2014. If analysis compared TAV replacement (TAVR) with SAVR from the 1970s, then the authors would have a valid argument; but that is not the case in this analysis. Furthermore, patients undergoing TAVR and SAVR had an almost identical case selection committee, inclusion and exclusion criteria, event adjudication committee, echocardiographic core laboratory, and clinical sites (to keep 2 two fruit salads tasting roughly the same).

We also pose a question to the authors: When a new version of SAVR valve is introduced for clinical use, is a randomized trial performed to show superiority? No. In the past 12 years, more than 4 new surgical valves have been introduced worldwide with none of them approved or used widely under the auspices of a randomized trial. A retrospective analysis or prospective single-arm registry was performed and reported.

The authors argue that the surgical group was defined according to the as-treated principle, potentially including the higher-risk subgroup of patients initially allocated to TAVR who received surgery, biasing the results in favor of TAVR. A close look at Figure 1 in the article by Thourani and colleagues $^{2}$ demonstrates that this is not true. From the patients who were randomly allocated to surgery in the Placement of Aortic Transcatheter Valves 2A trial, 68 patients withdrew before treatment, 4 were ineligible due to aortic calcification, and 5 died before treatment, leaving 944 patients (ie, the as-treated group who initiated surgery). None of these patients were TAVR patients who received surgery. The reason for choosing this group was to compare TAVR using a Sapien 3 valve with patients who actually received SAVR, rather than patients who did not undergo a procedure whatsoever. The median Society of Thoracic Surgeons score was $5.2 \%$ in the TAVR group and $5.4 \%$ in the SAVR group. The authors note the statistically different Society of Thoracic Surgery scores between the 2 groups as a significant limitation. We used propensity score analysis to address and adjust for this and other differences between the groups.

The authors question how a small change like adding a skirt to the Sapien 3 valve can translate into hard clinical end points. The authors should consider in addition to the skirt a more reliable positioning and deployment of the Sapien 3 valve due to the balloon inflation mechanism; the more maneuverable delivery system, which minimizes interaction with the aortic wall and aids in coaxial positioning; and smaller sheaths through which the valve can be introduced, leading to nearly $90 \%$ use of the transfemoral route. One can imagine that the so-called simple addition of a skirt, which reduces paravalvular leak, may lead to decreased need for dilation of the valve, with implications related to aortic root ruptures and strokes. 